\title{
Entropy Based Multi-criteria Decision Making Method under Fuzzy Environment and Unknown Attribute Weights
}

\author{
Harish Garg ${ }^{1 *}$, Nikunj Agarwal ${ }^{2}$ and Alka Choubey ${ }^{2}$
}

${ }^{1}$ School of Mathematics and Computer Applications, Thapar University, Patiala 147004, India

${ }^{2}$ Department of Mathematics, Jaypee Institute of Information and Technology, Noida-201307, U.P, India

\begin{abstract}
The main purpose of this paper is to investigate the multi-criteria decision making (MCDM) under the completely unknown attributes weights. As the information collected from the various resources related to different criteria for assessing the best alternatives is always imprecise in nature. Thus, to handle the impreciseness in the data, fuzzy set theory has been used during the analysis and representation each attribute in the form of triangular fuzzy numbers. Moreover, the attribute weight vectors, used for aggregating the decision maker's preferences, are found by using an entropy function. Finally, a house selection example, has been taken for demonstrating the approach.
\end{abstract}

Keywords: Multi-criteria decision making; Entropy functions; Attribute weights; Linguistic variables

\section{Introduction}

Decision making is one of the most significant and omnipresent human activities in business, service, manufacturing, selection of products, etc. As it is quite common that the different attributes play a significant role during the selection of the best alternatives among the existing ones. During their selection, one of the significant issues is to find the proper standardized weight vectors which are useful for aggregating the different decision maker's preferences. Technique for order preference by similarity to an ideal solution (TOPSIS), known as a classical multiple attribute decision making (MADM) method, has been developed by Hwang and Yoon [1] for solving the MADM problem. It is based on the idea that the chosen alternative should have the shortest distance from the positive ideal solution, and, on the other side, the farthest distance from the negative ideal solution. Traditionally, it has been generally assumed that all the information which access the alternative in terms of criteria and their corresponding weights are expressed in the form of crisp numbers [1]. In spite of its popularity, the method is often criticized for its inability to adequately handle the inherent uncertainty and imprecision associated with the mapping of a decision-maker's perception to crisp numbers.

However, in many cases the preference model of the human decision maker is uncertain and fuzzy and it is relatively difficult crisp numerical values of the comparison ratios to be provided by subjective perception. The decision maker may be subjective and uncertain about his level of preference due to incomplete information or knowledge, inherent complexity and uncertainty within the decision environment, lack of an appropriate measure or scale. Thus, if the assessment values are known to have various types of vagueness/imprecision or subjectiveness, then the classical decision making techniques are not useful for such problems. In the past few years, numerous attempts have been carried out to handle these issues and hence researchers have extended classicial multi-criteria decision making problem into the fuzzy multi-criteria decision making problem by suitably expressed the input of the decisions in the form of fuzzy numbers rather than crisp numbers. For instance, Tsaur et al. [2] first convert a fuzzy MADM problem into a crisp one via centroid defuzzification and then solve the nonfuzzy MADM problem using the TOPSIS approach. Chen and Tzeng [3] transform a fuzzy multiple criteria decision making (MCDM) problem into a nonfuzzy MADM using fuzzy integral. Instead of using distance, they employ a grey relation grade to define the relative closeness of each alternative. Chu [4] and [5] also changes a fuzzy MADM problem into a crisp one and solves the problem using the TOPSIS approach. Differing from the others, he first derives the membership functions of all the weighted ratings in a weighted normalization decision matrix using interval arithmetic of fuzzy numbers and then defuzzifies them into crisp values using the ranking method of mean of removals. Chen [6] extends the TOPSIS approach to fuzzy group decision making situations by defining a crisp Euclidean distance between any two fuzzy numbers. Triantaphyllou and Lin [7] develop a fuzzy version of the TOPSIS approach based on fuzzy arithmetic operations, resulting in a fuzzy relative closeness for each alternative. Hsu and Chen [8] discuss an aggregation of fuzzy opinions under group decision making. Li [9] proposes a simple and efficient fuzzy model to deal with multi-judges/MADM problems in a fuzzy environment. Liang [10] incorporates fuzzy set theory and the basic concepts of positive ideal and negative ideal points, and extends MADM to a fuzzy environment. Raj and Kumar [11] also used the maximizing set and minimizing set to rank alternatives presented by approximate trapezoidal fuzzy numbers. In Chen's work [6], he constructed the normalized values for the ideal solution and negative ideal solution on criteria. The normalized values for the ideal solution and negative ideal solution on criteria are always $(1,1,1)$ and $(0,0,0)$ respectively. $(1,1,1)$ and $(0,0,0)$ are extreme values which are possibly far from away true max and min values, so the extreme values could not represent the max and min values of TOPSIS. Beside the disadvantage of extreme values, the weighted ratings on criteria in Chen's work are presented by triangular fuzzy numbers as ratings, and weights are triangular fuzzy numbers. In fact, the multiplication between two triangular fuzzy numbers should be an approximate triangular fuzzy number, not a triangular fuzzy number. Thus, the computation of Chen

*Corresponding author: Harish Garg, School of Mathematics and Computer Applications, Thapar University, Patiala 147004, India, Tel: +91-86990-3114; E-mail: harishg58iitr@gmail.com

Received March 11, 2015; Accepted April 08, 2015; Published April 30, 2015

Citation: Garg H, Agarwal N, Choubey A (2015) Entropy Based Multi-criteria Decision Making Method under Fuzzy Environment and Unknown Attribute Weights. Global J Technol Optim 6: 182. doi:10.4172/2229-8711.1000182

Copyright: ( $2015 \mathrm{Garg} \mathrm{H}$, et al. This is an open-access article distributed under the terms of the Creative Commons Attribution License, which permits unrestricted use, distribution, and reproduction in any medium, provided the original author and source are credited. 
is very simple, but the weighted ratings could not express approximate triangular fuzzy numbers. To avoid these above problems, Wang and Lee [12] proposed a fuzzy multiple-criteria group decision-making method called fuzzy TOPSIS in a fuzzy environment. In it, they used max and min operations for finding the negative and ideal solutions. Aprt from that, various authors [13-20] have addressed the problems of MCDM under the fuzzy environment.

Moreover, from the survey, it has been observed that the final ranking order of alternatives highly depends on the attribute weights and hence the proper assessment of the attributes weights play a dominant role in the decision-making process. Based on the information acquisition, the attribute weight in multi-criteria decision making is classified as subjective as well as objective. The former ones are determined by preference information on the attributes as given by the decision-maker while the latter one are determined by the decisionmaking matrix. The Shannon entropy method $[1,21]$ is one of the most famous approach for determining the objective attribute weights, which express the relative intensities of attribute importance to signify the average intrinsic information transmitted to the decision maker. Thus, it is an important task for finding the proper attribute weights which will help the decision maker for obtaining the efficient decision under a reasonable time.

Therefore, this paper continues to study the multi-criteria decision making problems under the fuzzy environment with complete unknown attributes weight information. In the present paper, we extend the approach of TOPSIS to develop a methodology for solving the MADM problem in fuzzy environment. Considering the fuzziness in the decision data, linguistic variables are used to rate the each alternative with respect to each criterion and then convert the decision matrix into the fuzzy decision matrix. The unknown attributes weights of each criteria have been given by using the fuzzy entropy measurement and assessment of the different preferences for rating the attributes are represented in the form of linguistic variables in terms of triangular fuzzy numbers instead of crisp numbers. Based on these attributes weight and fuzzy decision matrix we construct a weighted normalized fuzzy decision matrix once the decision makers' fuzzy ratings have been pooled. The lower bound value of alternatives has been designed to obtain the distance value of the corresponding alternatives for detecting the fuzzy positive ideal solution (FPIS) and the fuzzy negative ideal solution (FNIS). Finally, a closeness coefficient is defined for each alternative, to determine the rankings of all alternatives. The higher value of closeness coefficient indicates that an alternative is closer to FPIS and farther from FNIS simultaneously.

The rest of the manuscript is summarized as follows. Section 2 discusses the basic primilaries related to fuzzy set theory. Section 3 describes the entropy based method for MCDM. A numerical example is utilized to illustrate the MCDM applicability of the proposed method in section 4. Finally, some concrete conclusion drawn is presented in Section 5 .

\section{Basic primilaries related to fuzzy set theory}

Fuzzy set theory: Fuzzy set [22] is treated as a generalization of the classical set theory where the membership functions are only treated by their belonginess or not. In fuzzy set theory, this relaxation is overlapped with the condition that it allows the partial membership between 0 and 1 also. Based on it, the fuzzy set $A_{i}^{\sim}$ in the universe of discourse $U$ is defined as the set of ordered pairs $A_{i}^{\sim}=\left\{\left\langle x, \mu_{A^{-}}(x)>\right| x \epsilon U\right\}$; where $\mu_{A^{-}}(x)$ represents the membership function of $x$ in the fuzzy set $\tilde{A_{i}}$ such that $0 \leq \mu_{A^{A}}(x) \leq 1$.

\section{Convex and normal set}

A fuzzy set is said to be convex fuzzy set if the following inequality holds for two points $x_{1}, x_{2} \epsilon U$

$$
\mu_{\tilde{A}}\left(\lambda x_{1}+(1-\lambda) x_{2}\right) \geq \min \left[\mu_{\tilde{A}}\left(x_{1}\right), \mu_{\tilde{A}}\left(x_{2}\right)\right]
$$

and if there is atleast one element $x \epsilon U$ such that $\mu_{A}(x)=1$ then that set is called as normal set.

\section{Fuzzy numbers}

A triangular fuzzy number $A \tilde{=}<a_{1}, a_{2}, a_{3}>$ such that $a_{1} \leq a_{2} \leq a_{3}$ and all $a_{1} \leq a_{2} \leq a_{3} \epsilon R$ is a convex and normal fuzzy set of the real line $R$ such that $\mu_{A}(x)$ is a piecewise continuous and there exist at least one $x_{0}$ $\epsilon U$ such that $\mu_{A^{r}}\left(x_{0}\right)=1$ and their corresponding membership function is given by

$$
\mu_{\tilde{A}}(x)= \begin{cases}\frac{x-a_{1}}{a_{2}-a_{1}} & ; a_{1} \leq x<a_{2} \\ 1 & ; x=a_{2} \\ \frac{a_{3}-x}{a_{3}-a_{2}} & ; a_{2} \leq x<a_{3}\end{cases}
$$

Here, $a_{1}$ represents the smallest possible bound, $a_{2}$ represents the modal (crisp) value and $a_{3}$ represents the largest possible value that describes the fuzzy events.

\section{Distance between the fuzzy triangular numbers}

Let $\tilde{a=}\left(a_{1}, a_{2}, a_{3}\right)$ and $\tilde{b}=\left(b_{1}, b_{2}, b_{3}\right)$ be two triangular fuzzy numbers, then the distance between them is computed as

$$
d(\tilde{a}, \tilde{b})=\sqrt{\frac{1}{3}\left[\left(a_{1}-b_{1}\right)^{2}+\left(a_{2}-b_{2}\right)^{2}+\left(a_{3}-b_{3}\right)^{2}\right]}
$$

\section{Linguistic variables}

The fuzzy theory provides the mechanism for representing the linguistic constructs such as "many", "low", "medium", "few", etc. In general, fuzzy logic provides an inference structure that enables appropriate human reasoning capabilities. A linguistic variable is characteristic by $(X, T, U, M)$ where $X$ is the name of the variable, $T$ is the set of the terms of $X, U$ is the universe of discourse and $M$ is the semantic rule for associating each term with a fuzzy set in $U$ Here, in this paper, triangular fuzzy numbers have been used for representing the linguistic variable in the scale of 0 to 1 for rating the criteria and the alternative. These various linguistic variables are represented as very poor (VP), poor (P), medium poor (MP), fair (F), medium good (MG), $\operatorname{good}(\mathrm{G})$, and very good (VG).

\section{Entropy Based Multi-criteria Decision making Approach}

The multi-criteria decision making $(\mathrm{MCDM})$ is a very practical method in the real world, and have very significant effect on both theory and practical. The aim of the MCDM is to find the best alternative among the given criteria. In this section, we shall investigate the multiple criteria decision making problems under fuzzy environment and the attribute weight to each criteria is computed based on defining their corresponding entropy method. For a MCDM problem, assume that a set of option/alternatives $A_{1}, A_{2}, \ldots A_{m}$ to be considered under the set of $n$ evaluation criteria $\mathrm{C}_{n}, C_{2}, \ldots C_{m}$. Assume that $w_{j}$ be the weight of criteria $C_{j}$ where $w_{j} \in[0,1], \sum_{j=1}^{n} w_{j}=1$. Let there be 
Citation: Garg H, Agarwal N, Choubey A (2015) Entropy Based Multi-criteria Decision Making Method under Fuzzy Environment and Unknown Attribute Weights. Global J Technol Optim 6: 182. doi:10.4172/2229-8711.1000182

a prioritization between the attributes expressed by the linear ordering $C_{1} \succ C_{2} \succ \ldots \succ C_{n}$ (indicating that the attribute $C_{1}$ has a higher priority than $C_{j}$, if $i<j$ ). Let $G_{i j}$ is the performance rating of the alternative $A_{i}$ $(i=1,2, \ldots m)$. under criteria $C_{j}(j=1,2, \ldots, n)$ which are expressed in the form of fuzzy decision matrix and $a_{i j}=\left(a_{i j}, b_{i j}, c_{i j}\right)$ be an attribute value provided by the decision maker for the alternative $A_{i}$ with respect to the attribute $C_{i}$, where $a_{i j}$ represents the lower value, $b_{i j}$ represents the modal value and $c_{i j}$ represents the upper value of the attribute and then the corresponding fuzzy decision matrix is expressed as

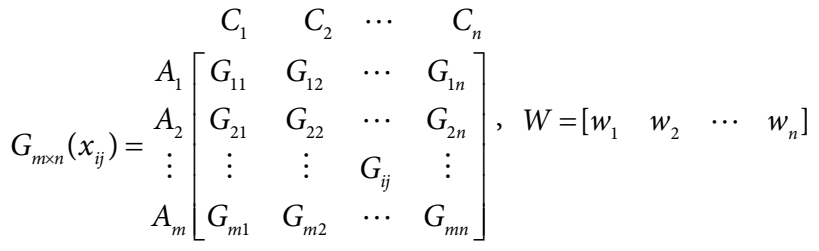

Then we have the following decision making method which consists of the following steps for evaluation.

Step 1: Assignments of rating to the criteria and the alternatives: - The performance rating of each decision maker $D_{k}(k=1,2, \ldots K)$ for each alternative $A_{j}(j=1,2, \ldots m)$ with respect to criteria $C_{j}(j=1,2, \ldots n)$ are assessed in the form of triangular fuzzy numbers and their corresponding matrix is denoted by $\tilde{R}_{k}=\left(a_{i j}^{k}, b_{i j}^{k}, c_{i j}^{k}\right)(i=1, \ldots, m ; j=1, \ldots n ; k=1, \ldots, K)$ . Based on these fuzzy rating, the aggregating fuzzy rating $\tilde{x_{i j}}$ of alternatives with respect to each criteria are given by $\tilde{x_{i j}}=\left(\begin{array}{lll}a_{i j} & b_{i j} & c_{i j}\end{array}\right)$ where $a_{i j}=\sum_{k=1}^{K} \frac{a_{i j}^{k}}{K}, b_{i j}=\sum_{k=1}^{K} \frac{b_{i j}^{k}}{K}$ and $c_{i j}=\sum_{k=1}^{K} \frac{c_{i j}^{k}}{K}$

Step 2: Normalize the fuzzy decision matrix: Normalize each attribute value $x_{i j}(i=1,2 \ldots m ; j=1,2 \ldots n)$ in the matrix $R_{i k}^{\sim}$ into the corresponding element in the matrix called normalized fuzzy decision matrix $R_{i}^{\sim}=\left[\tilde{r}_{i i j}\right]_{m \times n}$, where $\tilde{r}_{i j}=\left(\frac{a_{i j}}{c_{j}^{+}}, \frac{b_{i j}}{c_{j}^{+}}, \frac{c_{i j}}{c_{j}^{+}}\right)$and $c_{j}^{+}=\max _{i} c_{j} \quad$ (benefit criteria); $\tilde{r}_{i j}=\left(\frac{a_{j}^{-}}{c_{i j}}, \frac{a_{j}^{-}}{b_{i j}}, \frac{a_{j}^{-}}{a_{i j}}\right)$ and $a_{j}^{-}=\min _{i} a_{i j} \quad$ (cost criteria).

Step 3: Determination of the entropy matrix: Based on the constructed normalized matrix $R_{i}^{\sim}$ in step 2 , the weights corresponding to each attribute $w_{j}(j=1,2, \ldots n)$ can be calculated $w_{j}=\xi_{j} / \sum_{j=1}^{n} \xi_{j}$ where $\xi_{j}=1-E\left(C_{j}\right)$ is called the degree of divergence and $E\left(C_{j}\right)$ is the information entropy [1] of attribute $C_{j}$ which is calculated as

$$
E\left(C_{j}\right)=-\kappa \sum_{i=1}^{m}\left[\mu_{i j} \ln \left(\mu_{i j}\left(C_{j}\right)\right)+\left(1-\mu_{i j}\left(C_{j}\right)\right) \ln \left(1-\mu_{i j}\left(C_{j}\right)\right)\right],
$$

where, $\kappa$ is constant such that $0 \leq E\left(C_{j}\right) \leq 1$.

Step 4: Compute the fuzzy PIS and fuzzy NIS: The fuzzy PIS and fuzzy NIS of the alternatives are calculated as $A^{+}=\left(\tilde{p}_{1}^{+}, \tilde{p}_{2}^{+}, \ldots, \tilde{p}_{n}^{+}\right)$, where $\tilde{p}_{j}^{+}=\max \left\{v_{i j}\right\} ; A^{-}=\left(\tilde{p}_{1}^{-}, \tilde{p}_{2}^{-}, \ldots, \tilde{p}_{n}^{-}\right)$, where $\tilde{p}_{j}^{-}=\min \left\{v_{i j}\right\}$; where $\tilde{v_{i j}}=\tilde{r_{i i j}} \cdot \tilde{w_{i j}}$ is the normalized weighted matrix $V_{i}^{r}=\left[\tilde{v_{i j}}\right]_{m \times n}$.

Step 5: Compute distance from each alternative: Let $d_{i j}^{-}$and $d_{i j}^{+}$ be distanced from alternatives to the ideal solution (or negative ideal solution) i.e. distance of $v_{i j}$ to $p_{j}^{-}$and $p_{j}^{+}$respectively, therefore, $d_{i j}^{-}=d\left(\tilde{v}_{i j}, \tilde{p}_{j}^{-}\right)$and $d_{i j}^{+}=d\left(\tilde{v}_{i j}, \tilde{p}_{j}^{+}\right)$. Let $D_{i}^{-}=\sum_{j=1}^{n} w_{j} \otimes d_{i j}^{-}$and $D_{i}^{+}=\sum_{j=1}^{n} w_{j} \otimes d_{i j}^{+}$for $i=1,2, \ldots, m$ be representing their corresponding weighted distance. Thus, a weighted distance of $d_{i}$ can be expressed by $\left[D_{i}^{-}, D_{i}^{+}\right]$. Let $L D^{-}=\min \left(\left\{D_{1}^{-}, D_{2}^{-}, \ldots, D_{m}^{-}\right\}\right)$, $U D^{-}=\max \left(\left\{D_{1}^{-}, D_{2}^{-}, \ldots, D_{m}^{-}\right\}\right), \quad L D^{+}=\min \left(\left\{D_{1}^{+}, D_{2}^{+}, \ldots, D_{m}^{+}\right\}\right) \quad$ and $U D^{+}=\max \left(\left\{D_{1}^{+}, D_{2}^{+}, \ldots, D_{m}^{+}\right\}\right)$be the fuzzy minimum and maximum values of $D_{i}^{-}$and $D_{i}^{+}$. Therefore, $d_{i}^{-}$denote the distance from $\left[D_{i}^{-}, D_{i}^{+}\right]$to $\left[L D^{-}, U D^{+}\right]$and $d_{i}^{+}$denote the distance from $\left[D_{i}^{-}, D_{i}^{+}\right]$to $\left[U D^{-}, L D^{+}\right]$. Thus, $d_{i}^{+}=d\left(D_{i}^{-}, L D^{-}\right)+d\left(D_{i}^{+}, U D^{+}\right)$and $d_{i}^{-}=d\left(D_{i}^{-}, U D^{-}\right)+d\left(D_{i}^{+}, L D^{+}\right)$for $i=1,2, \ldots, m$, where $d\left(\tilde{a_{i}} \tilde{b_{i}}\right)$ is the distance measurement between two fuzzy numbers $\tilde{a_{i}}$ and $\tilde{b_{i}}$.

Step 6: Compute the closeness coefficient $\left(C C_{i}\right)$ of each alternative: The closeness coefficient $\left(C C_{i}\right)$ represents the distances to the FPIS and FNIS simultaneously and hence closeness coefficient of each alternative is calculated as: $C C_{i}=\frac{d_{i}^{-}}{d_{i}^{-}+d_{i}^{+}}, i=1,2, \ldots \ldots, m$

Step 7: Rank the alternatives. Finally, rank all the alternatives $A$ according to the closeness coefficient in decreasing order..

\section{Illustrative Example}

In order to demonstrate the applications of the develop methodology, we will consider an example where consists of 3 houses $H_{1}, H_{2}, H_{3}$ examined by the four experts under fuzzy environment for the selection of houses against the 4 criteria or parameters $C_{1^{\prime}}, C_{2^{\prime}} C_{3^{\prime}}$ $\mathrm{C}_{4}$ defined as;
$C_{1}$ :expensive,
$\mathrm{C}_{2}$ : Beautiful,
$C_{3}$ :In good repair,
$\mathrm{C}_{4}$ :In good surrounding

In order to take the decision related to these houses, four experts give the linguistic performance ratings, VP, P, MP, F, MG, G, and VG, in terms of fuzzy numbers which are as follows:

Very Poor $(\mathrm{VP})=(0,0,0.2)$,

Poor $(\mathrm{P})=(0,0.2,0.4)$,

Most Poor $(\mathrm{MP})=(0.2,0.4,0.5)$,

Fair $(\mathrm{F})=(0.4,0.5,0.6)$,

Most Good $(\mathrm{MG})=(0.5,0.6,0.8)$,

$\operatorname{Good}(\mathrm{G})=(0.6,0.8,1.0)$,

Very Good $(\mathrm{VG})=(0.8,1,1)$

of the three houses. These linguistic performance ratings are shown in Table 1 . Thus, by using step 1 of the proposed approach, the average rating of the three houses by the experts in terms of fuzzy numbers are summarized in Table 2.

Based on the entropy function given in Eq. (1), the attribute weights are computed as $w_{1}=0.2665 ; w_{2}=0.2578 ; w_{3}=0.2185 ; w_{4}=0.2570$ and hence FPIS of each alternative are $p_{1}^{+}=(0.625,0.80,0.95)$, $p_{2}^{+}=(0.65,0.85,1.0), \quad p_{3}^{+}=(0.7,0.9,1.0), \quad p_{4}^{+}=(0.75,0.95,1.0) \quad$ while FNIS are $\quad p_{1}^{-}=(0.45,0.55,0.7), \quad p_{2}^{-}=(0.60,0.75,0.90), \quad p_{3}^{-}=(0.425,0.525,0.65)$, $p_{4}^{-}=(0.475,0.575,0.75)$. By using this information, the minimum and maximum bounds are $U D^{+}=0.1206, L D^{+}=0.06957, U D^{-}=0.1390$ and $L D^{+}=0.1157$. Hence, the distance of each alternative from its FPIS and FNIS are $d_{1}^{+}=0, d_{1}^{-}=0.0482, d_{2}^{+}=0.0214, d_{2}^{-}=0.0268, d_{3}^{+}=0.0482$ 
Citation: Garg H, Agarwal N, Choubey A (2015) Entropy Based Multi-criteria Decision Making Method under Fuzzy Environment and Unknown Attribute Weights. Global J Technol Optim 6: 182. doi:10.4172/2229-8711.1000182

\begin{tabular}{|c|c|c|c|}
\hline $\boldsymbol{G}_{i j}$ & $\boldsymbol{H}_{1}$ & $\boldsymbol{H}_{2}$ & $\boldsymbol{H}_{3}$ \\
\hline$C_{1}$ & $\{\mathrm{MG}, \mathrm{G}, \mathrm{G}, \mathrm{VG}\}$ & $\{\mathrm{VG}, \mathrm{G}, \mathrm{MG}, \mathrm{MG}\}$ & $\{\mathrm{MG}, \mathrm{F}, \mathrm{MG}, \mathrm{F}\}$ \\
\hline$C_{2}$ & $\{\mathrm{MG}, \mathrm{VG}, \mathrm{G}, \mathrm{MG}\}$ & $\{\mathrm{G}, \mathrm{G}, \mathrm{VG}, \mathrm{G}\}$ & $\{\mathrm{G}, \mathrm{VG}, \mathrm{G}, \mathrm{G}\}$ \\
\hline$C_{3}$ & $\{\mathrm{~F}, \mathrm{~F}, \mathrm{~F}, \mathrm{MG}\}$ & $\{\mathrm{VG}, \mathrm{G}, \mathrm{MG}, \mathrm{G}\}$ & $\{\mathrm{VG}, \mathrm{VG}, \mathrm{G}, \mathrm{G}\}$ \\
\hline$C_{4}$ & $\{\mathrm{VG}, \mathrm{G}, \mathrm{VG}, \mathrm{VG}\}$ & $\{\mathrm{F}, \mathrm{MG}, \mathrm{MG}, \mathrm{MG}\}$ & $\{\mathrm{MG}, \mathrm{MG}, \mathrm{G}, \mathrm{MG}\}$ \\
\hline
\end{tabular}

Table 1: Linguistic performance rating of the three houses.

\begin{tabular}{|c|c|c|c|}
\hline $\boldsymbol{G}_{i j}$ & $\boldsymbol{H}_{1}$ & $\boldsymbol{H}_{2}$ & $\boldsymbol{H}_{3}$ \\
\hline $\mathrm{C}_{1}$ & $(0.625,0.80,0.95)$ & $(0.60,0.75,0.90)$ & $(0.45,0.55,0.70)$ \\
\hline $\mathrm{C}_{2}$ & $(0.600,0.75,0.90)$ & $(0.65,0.85,1.00)$ & $(0.65,0.85,1.00)$ \\
\hline $\mathrm{C}_{3}$ & $(0.425,0.525,0.65)$ & $(0.625,0.80,0.95)$ & $(0.70,0.90,1.00)$ \\
\hline $\mathrm{C}_{4}$ & $(0.75,0.95,1.00)$ & $(0.475,0.575,0.75)$ & $(0.525,0.65,0.85)$ \\
\hline
\end{tabular}

Table 2: Average rating of three houses on four criteria.

and $d_{3}^{-}=0$. Thus, the evaluated results about the houses are computed by using the closeness coefficient $H_{i}=\frac{d_{i}^{-}}{d_{i}^{-}+d_{i}^{+}}$as $H_{1}=1, H_{2}=0.5560$, $H_{3=} 0$. Therefore, ranking of the houses are $H_{1} \succ H_{2} \succ H_{3}$ and hence $H_{1}$ is the most desirable one while $\mathrm{H}_{3}$ is the least one. Thus, $H_{1}$ will be the best house to be purchased.

\section{Discussion}

As the attribute weights are the most important parameter for the decision maker for accessing the best alternative. So, in order to compare the proposed results with the existing techniques $[6,12,17]$, the corresponding approaches have been implemented here for the considered data with the attribute weights $w_{1}=0.2665 ; w_{2}=0.2578$; $w_{3}=0.2185 ; w_{4}=0.2570$ and found that the proposed results coincides with the ones shown one shown in $[6,12,17]$. Thus, it has been concluded from the aforementioned results that the proposed decision making method can be suitably utilized to solve the multiple and decision making problem with completely unknown attribute weights.

\section{Conclusion}

In the present work, an approach for selecting the best alternative under the different selection or criteria is implemented. The approach has been illustrated through a case study of selected the house against the different criteria. The preferences of the various alternatives are represented in terms of linguistic variation as a triangular fuzzy number. As the key issues of decision making with incomplete weight information are to find the proper way to derive the weight vector and to aggregate the decision makers' preferences. For this, entropy based method has been proposed for finding the attribute weights corresponding to each objective. Based on their weights, all the individual decision makers' opinion for rating the candidate are aggregated using the fuzzy positive and negative ideal solutions. A ranking of the different alternatives has been done by using the closeness coefficient and based on that the best alternative is selected. The proposed method provides us with a useful way for dealing with multi-criteria fuzzy decision making problems within attribute weights. Although, we have focused on the MCDM in fuzzy environment in this paper, we have extended the present work in the direction of taking intuitionistic fuzzy numbers and intervalvalued intuitionistic fuzzy numbers. The work can also be extended to devise a suitable optimization model for deriving the attribute weights corresponding to alternative and hence best alternative is chosen according to them. Finally, different operators may use to aggregate the different alternatives and hence based on that ranking of the different alternative done.

\section{References}

1. Hwang CL, Yoon K (1981) Multiple Attribute Decision Making-Methods and Applications: A State-of-the-Art Survey, Springer-Verlag, New York.

2. Tsaur SH, Chang TY, Yen $\mathrm{CH}$ (2002) The evaluation of airline service quality by fuzzy MCDM. Tourism Management 23: 107-115.

3. Chen MF, Tzeng GH (2004) Combining grey relation and TOPSIS concepts for selecting an expatriate host country. Mathematical and Computer Modeling 40: $1473-1490$.

4. Chu TC (2002) Facility location selection using fuzzy TOPSIS under group decisions. International Journal of Uncertainty, Fuzziness and KnowledgeBased Systems 10: 687-701.

5. Chu TC (2002) Selecting plant location via a fuzzy TOPSIS approach. The International Journal of Advanced Manufacturing Technology 20: 859-864.

6. Chen CT (2000) Extension of the TOPSIS for group decision-making under fuzzy environment. Fuzzy Sets and Systems 114: 1-9.

7. Triantaphyllou E, Lin CT (1996) Development and evaluation of five fuzzy multi attribute decision-making methods. International Journal of Approximate Reasoning 14: 281-310.

8. Hsu HM, Chen CT (1996) Aggregation of fuzzy opinions under group decision making. Fuzzy Sets and Systems 79: 279-285.

9. Li DF (2005) Multi attribute decision making models and methods using intuitionistic fuzzy sets. Journal of Computer and System Sciences 70: 73-85.

10. Liang GS (1999) Fuzzy MCDM based on ideal and anti-ideal concepts. European Journal of Operational Research 112: 682-691.

11. Raj PA, Kumar DN (1999) Ranking alternatives with fuzzy weights using maximizing set and minimizing set. Fuzzy Sets and Systems 105: 365-375.

12. Wang Y, Lee HS (2007) Generalized TOPSIS for fuzzy multiple-criteria group decision making. Computers and Mathematics with Applications 53: 1762-1772.

13. Mahdavi I, Mahdavi-Amiri N, Heidarzade A, Nourifar R (2008) Designing a mode of fuzzy TOPSIS in multiple criteria decision making. Applied Mathematics and Computations 206: 607-617.

14. Maniya KD, Bhatt MG (2013) A Selection of Optimal Electrical Energy Equipment Using Integrated Multi Criteria Decision Making Methodology. International Journal of Energy Optimization and Engineering 2: 101-116.

15. Oztaysi B, Isik M, Ercan S (2013) Multi-Criteria Decision Aid for Sustainable Energy Prioritization Using Fuzzy Axiomatic Design. International Journal of Energy Optimization and Engineering 2: 1-20.

16. Cavallaro F (2013) Assessment of Nuclear Energy Competiveness Using a Multi-Criteria Fuzzy Approach. International Journal of Energy Optimization and Engineering 2: 21-36.

17. Wang YJ, Lee HS, Lin K (2003) Fuzzy TOPSIS for multi-criteria decisionmaking. International Mathematical Journal 3: 367-379.

18. Saghafian S, Hejazi S (2005) Multi-criteria group decision making using a modified fuzzy TOPSIS procedure, Computational Intelligence for Modelling Control and Automation, 2005 and International Conference on Intelligent Agents, Web Technologies and Internet Commerce, International Conference on 2: $215-221$

19. Jiang Y, Fan Z, Ma J (2008) A method for group decision making with multigranularity linguistic assessment information. Information Sciences 178: 1098-1109.

20. Kaya T, Kahraman C (2011) Multi-criteria decision making in energy planning using a modified fuzzy TOPSIS methodology. Expert Systems with Applications 38: 6577-6585.

21. Klir GJ, Yuan B (2005) Fuzzy Sets and Fuzzy Logic: Theory and Application Prentice Hall of India Private Limited, New Delhi, India.

22. Zadeh LA (1965) Fuzzy sets. Information and Control 8: 338-353. 